\title{
CLINICAL EVALUATION OF THE INTRA-ARTICULAR INJECTIONS OF PLATELET RICH PLASMA VERSUS DEXTROSE PROLOTHERAPY FOR MANAGEMENT OF TEMPOROMANDIBULAR JOINT INTERNAL DERANGEMENT
}

\author{
Yasser Habaka ${ }^{1}$, Eman Elsharrawy ${ }^{2}$, Tamer Abdel Bari ${ }^{3}$, Abdelbadia bdelmabood ${ }^{4}$
}

DOI: $10.21608 /$ dsu.2021.24255.1028

Manuscript ID: DSU-2002-1028

\section{KEYWORDS}

Internal derangement, TMD's, prolotherapy, PRP injection.
- E-mail address: Yasserhabaka35@gmail.com

1. Assistant Lecturer oral and maxillofacial surgery, Oral Surgery Department, Faculty of Dentistry, October 6 University.

2. Professor of Anesthesiology Oral and Maxillofacial surgery department, Faculty of Dentistry, Suez Canal University

3. Professor of Oral and Maxillofacial Surgery, Faculty of Dentistry, Suez Canal University

4. Assistant Professor of Oral and Maxillofacial surgery, Faculty of Dentistry, Zagazig University

\begin{abstract}
Aim: The aim of the study was to evaluate the clinical effect of intra-articular injections of platelet rich plasma for management of TMJ internal derangement through comparative study with dextrose prolotherapy. Patients and Methods: included 87 joints in 45 adult patients complaining pain, clicking in the Temporo-mandibular joint (internal derangement), pre-operative MRI was done for all patients with further clinical, radiographic as well as TMJ examination. Patients were divided into three equal groups randomly. Group I: (study group) composed of 15 patients where $1 \mathrm{ml}$ of platelet rich plasma was injected intra-articular (once) Group II: (study group) composed of 15 patients where $1 \mathrm{ml}$ of platelet rich plasma was injected intra-articular (twice) with one month interval Group III: (control group) composed of 15 patients where dextrose prolotherapy was injected (4 times) at 2 weeks, 4 weeks and 6 weeks intervals over a total of 12 weeks. Clinical assessment was done including MIO, pain, deviation and clicking. Results: statistically analysis of this study showed that group I, III showed the highest MIO values as well as VAS scores in the 3,6 and 12 months, however group II showed the lowest MIO in the same follow up period. Conclusions: PRP and Prolotherapy are successful techniques for treatment of symptoms related to internal derangement of TMJ.
\end{abstract}

\section{INTRODUCTION}

Internal derangement of Temporo-mandibular joint (TMJ) in which disc displaced from its normal position with mandibular condyle and articular portion of temporal bone ${ }^{(1)}$. TMJ internal derangement is characterized by intra-articular disc displacement resulting in popping or clicking sounds. The displacement of articular disc does not always cause a mechanical obstruction. ${ }^{(2)}$ Those conditions may be with or without pain. Among causes are trauma, which results in a sudden disc displacement, or para functional habits which ends up in increased friction, degenerative changes of articular surfaces and gradual displacement of the disc ${ }^{(2)}$.

The aim of the present study was to evaluate the clinical effect of intra-articular injections of platelet rich plasma for management of temporomandibular joint internal derangement through comparative study with dextrose prolotherapy. 


\section{PATIENTS AND METHODS}

The present study included 87 joints in 45 adult patients suffering from internal derangement, selected from outpatient clinic, oral and maxillofacial surgery department in faculty of dentistry, Suez Canal University as well as October 6 university.

\section{Patient's selection}

\section{Inclusion criteria:}

1. Patients were diagnosed with internal derangement both clinically and through magnetic resonance imaging scan (MRI).

2. Experiencing unilateral or bilateral TMJ affection

3. Age (20-50) years.

\section{Exclusion criteria:}

1. Patients suffering from haematologic disorders (platelet function disorders \& anticoagulation therapy)

2. Renal and or hepatic insufficiency

3. Patients with prosthetic joint replacement

4. Patients allergic to any components of the injectable solution

\section{Patient's examination}

\section{A-History}

All patients were subjected to prepared medical questionnaire relied upon by the American Dental Association (ADA) (included in the appendix). Medical history was taken from all patients and documented in the patient's own chart.

Chief complaint \& history of chief complaint was taken from patient's own words.
Dental history and history of any surgical procedure previously done in the area of interest.

\section{B-Clinical Examination}

A comprehensive intraoral examination of oral structures was carried out in conjugation with the dental history

For the assessment of patients with TMJ ID, all patients were undergone;

\section{Visual Analogue Scale (VAS):}

The degree of pain and tenderness were recorded preoperatively and throughout the treatment to assess improvement

\section{Inter-incisal opening, figure (1a-b):}

a- Maximum inter-incisal opening $\rightarrow$ (AROM) Active Range of Motion:

The incisal opening was recorded in millimeters from upper and lower central incisors by asking the patients to open their mouth fully unassisted.

b- Assisted inter-incisal opening $\rightarrow$ (PROM) Passive Range of Motion:

The patients were asked to open to the point at which experienced pain by assisting their opening, then it was measured from the edges of upper and lower central incisors.

The measurements were always taken in the same point each visit for every patient from the same central incisor. 


\section{Deviation on opening:}

By observing the opening pattern for deviation, the mandible often deviates toward the affected side during opening due to muscles spasm or displaced disc.

\section{Muscles examination:}

The masticatory muscles were examined by palpation to determine the presence of tenderness or pain

a- Temporalis muscles $\rightarrow$ anterior, middle and posterior portion of the muscles were palpated during clenching

b- Masseter muscles $\rightarrow$ the muscles were palpated from origin to insertion when patients jaw was forcibly closed.

c- Lateral pterygoid muscles $\rightarrow$ the muscles were palpated medial to coronoid process and lateral to maxillary tuberosity.

\section{TMJ examination:}

The TMJ was examined by palpation through pre-auricular approach and the following were recorded:

a- Presence of tenderness or pain

b- Translation of disc during different mandibular movement

c- Joints sounds $\rightarrow$ presence or absence of clicking and weather it was:

i- Left, right or bilateral

ii- Painful / painless

iii- Consistent / intermittent

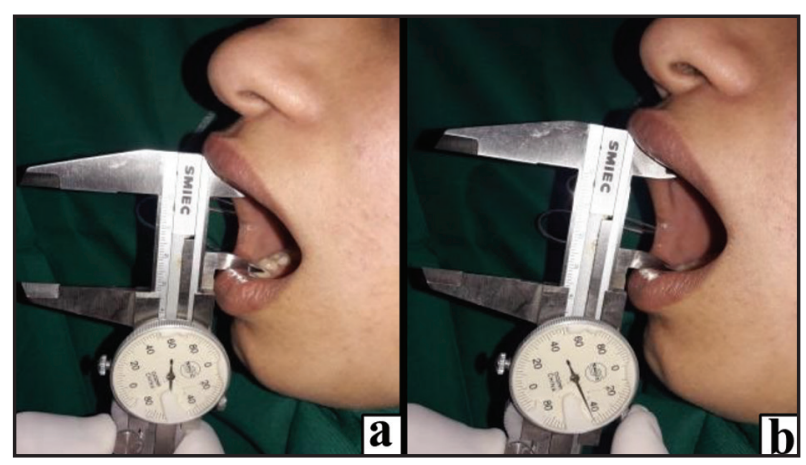

Fig. (1) a- Clinical photograph showing preoperative clinical assessment of TMJ , PROM in case no 1 group 3, 33 $\mathrm{mm}$, b- clinical photograph showing preoperative clinical assessment of TMJ, AROM in case no 1 group $3,36 \mathrm{~mm}$

\section{C-Radiographic Examination:}

Radiographic examination, including: - preoperative magnetic resonance imaging (MRI) was done for all patients in open and closed mouth position and interpretation was done:

A- Normal: the disc location was normal in open and closed mouth positions.

B- Displacement of disc with reduction: disc was displaced anteriorly in closed mouth position and in normal relation with condyle in open mouth position, figure (2,3a).

C- Displacement of disc without reduction: disc was displaced anteriorly in both closed and open mouth positions, figure (3b).

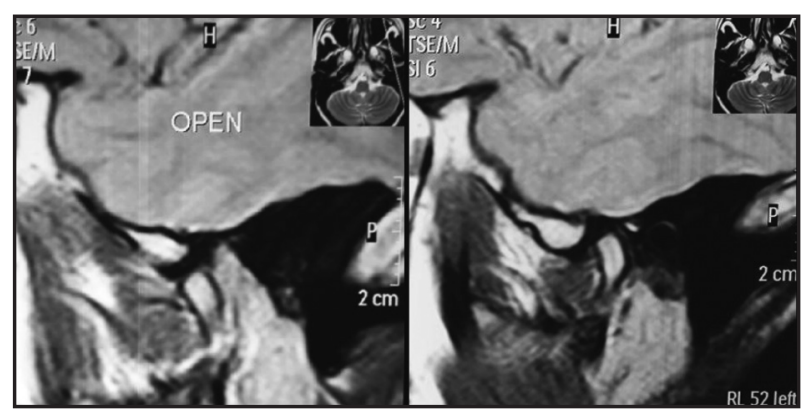

Fig. (2) Preoperative T1 MRI, sagittal view showing anterior displacement of the disc with reduction 


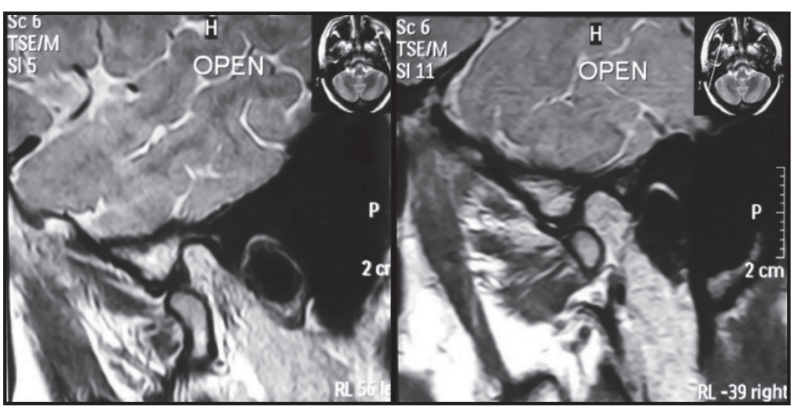

Fig. (3a-b): a- Preoperative T1 magnetic resonance imaging, sagittal view showing anterior disc displacement with reduction in right side. b- Anterior displacement of the disc without reduction in left side of same patient

\section{Study design}

Study were divided into three equal groups:

Group I: (study group) Composed of 15 patients where $1 \mathrm{ml}$ of platelet rich plasma was injected intra-articular (once)

Group II: (study group) Composed of 15 patients where $1 \mathrm{ml}$ of platelet rich plasma was injected intra-articular (twice) with one-month interval

Group III: (control group) Composed of 15 patients where dextrose prolotherapy was injected (4 times) at 2 weeks, 4 weeks and 6 weeks intervals over a total of 12 weeks.

\section{Patient preparation :}

Before administering injection, the skin over the target area is cleansed with appropriate antiseptic, draping around areas of injection with sterilized towels. Target areas marked with a washable felt-tip pen. Figure (4)

Group I: (study group) Platelet rich plasma (PRP): Composed of 15 patients where $1 \mathrm{ml}$ of platelet rich plasma was injected intra-articular (once).

\section{PRP preparation:}

Set was prepared for PRP collection, figure (5). $10 \mathrm{ml}$ blood collected and centrifuged for $6 \mathrm{~min}$ at $1500 \mathrm{rpm}$.

3 layers were obtained: the yellow top layer containing PPP; a pink middle layer containing PRP a red bottom layer containing red blood cells. The middle plasma layer (PRP) was the interest layer, Figure (7a-b).

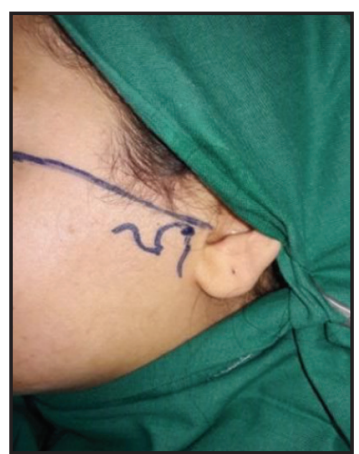

Fig. (4) Showing draping around areas of injection with sterilized towels and target areas marked with a washable felt-tip pen

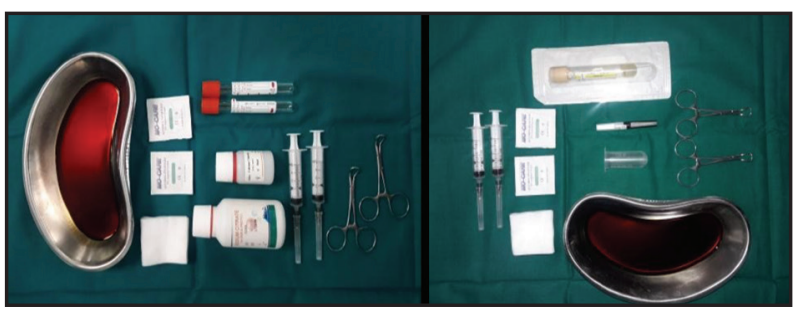

Fig. (5) Photograph showing prepared set for PRP collection

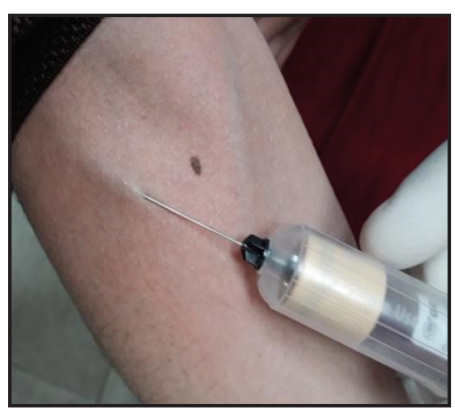

Fig. (6) PRP was taken from the antecubital vein in case no 1 group 1 


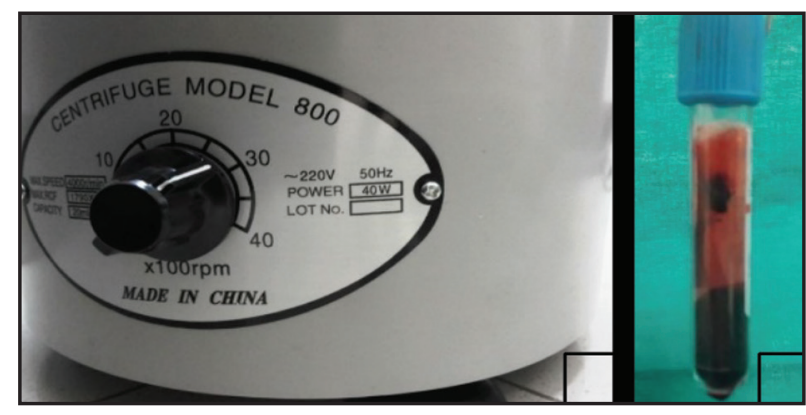

Fig. (7) a- clinical photograph showing blood centrifuge used for PRP formation, b- the collected PRP in the PRP citrated collector tube

PRP was then injected intra-articular, figure (8).

\section{Group II: (study group) Platelet rich plasma (PRP) :}

Composed of 15 patients where $1 \mathrm{ml}$ of platelet rich plasma was injected intra-articular (twice) at 1 month interval, figure (9).

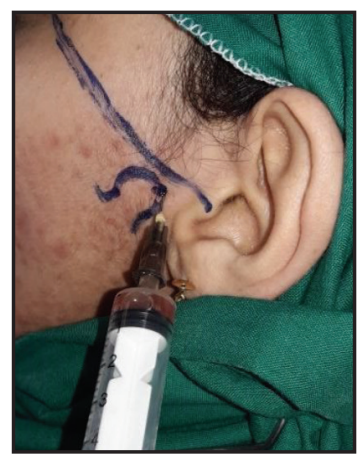

Fig. (8) Showing PRP injected intra-articular in case no 1 group I

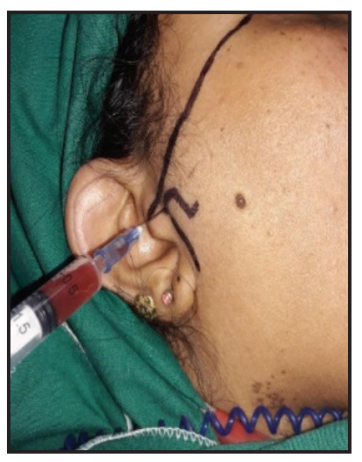

Fig. (9) Showing PRP injected intra-articular in case no 1 group II

\section{Group III: (control group) Prolotherapy technique:}

Composed of 15 patients where dextrose prolotherapy was injected (4 times) at 2 weeks, 4 weeks and 6 weeks intervals over a total of 12 weeks. This consists of $1.5 \mathrm{ml}$ from $25 \%$ dextrose and $1.5 \mathrm{ml}$ from $2 \%$ lidocaine into a $3-\mathrm{mL}$ syringe for each TMJ.

The result is a dextrose $12.5 \%$, figure (10) .

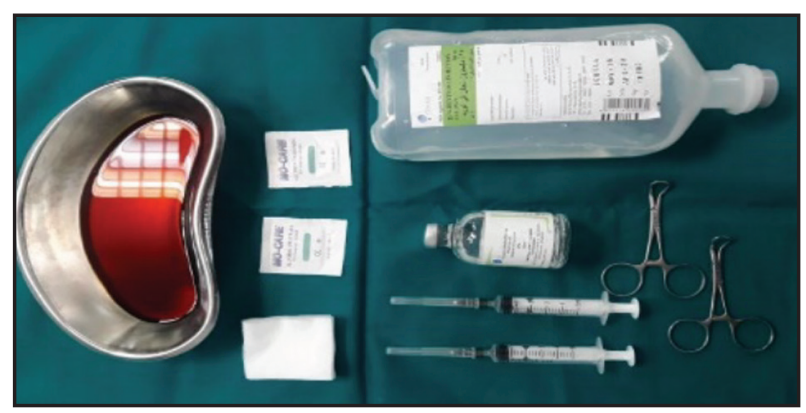

Fig. (10) Showing set was prepared for prolotherapy injection

A. Patient Posture and Head Position: The patient was preferably positioned supine or reclined.

B. Pre-injection Procedure and Selection of Injection Tools: $12.5 \%$ Dextrose which is the most commonly used prolotherapy solution, Before administering injection, the skin over the target area is cleansed with appropriate antiseptic, draping around areas of injection with sterilized towels. Then, the target areas were palpated and subsequently marked with a washable felt-tip pen. Figure (4)

C. Articular injection approach: The TMJ and facial structures are highly sensitive areas hence, injections should be atraumatic as possible.

Injections were made during maximal opening, $10 \mathrm{~mm}$ infront of the ear tragus and below the lateral cantho-tragal line by $2 \mathrm{~mm}$. Figure $(11 \mathrm{a}, \mathrm{b})$
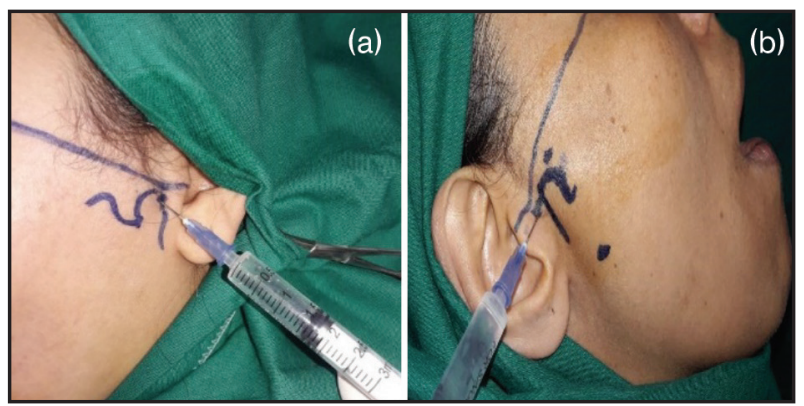

Fig. (11) (a) Showing points of needle insertion, case no 1 group II. (b) Showing points of needle insertion, case no 2 group II 
Anterior displacement of the disc of TMJ is the commonest, the first target area was Posterior joint space, which was palpated as the depression immediately anterior to tragus of ear during condylar translation forward and downward when patient opened his mouth.

A bite block was placed intraorally so that patient becomes unable to close his mouth during the procedure.

The injection needle directing medially and slightly anteriorly. Following aspiration, $1 \mathrm{~mL}$ of prolotherapy solution is deposited at this site.

Anterior disc attachment is the second target area, Prolotherapy injection solution play role to strength the tendinous muscle attachment, figure (12 a).

This target area was palpated as the depression just anterior to condyle during closure. This point was marked.

The next target was the tender muscle, by clenching teeth, the muscle prominent, and area that was most rigid, asked patients to relaxed their jaws to inject the final $\mathrm{ml}$. figure (12 b).

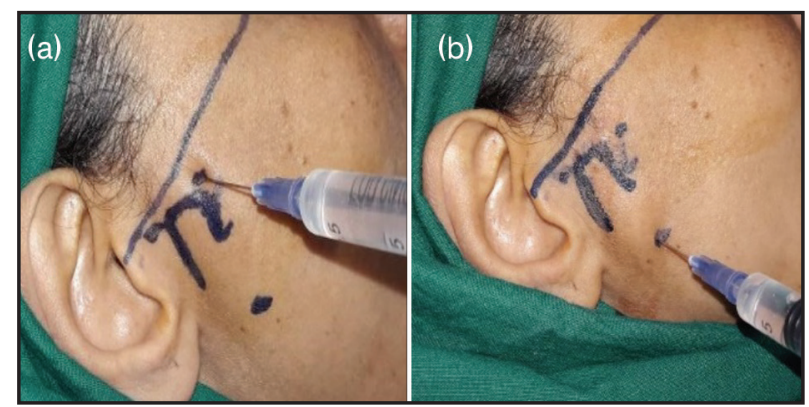

Fig. (12) (a) Showing a needle was inserted in the second target area, the anterior disc attachment, case no 2 group III. (b) Clinical photograph showing masseter muscle injection as the third target area in case no 2 group III.

The same procedure was repeated on opposite joint if it was affected, figure (13).

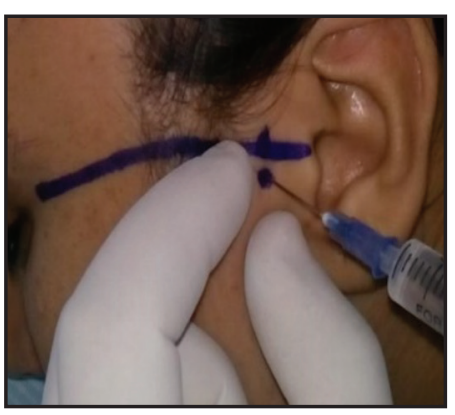

Fig. (13) Clinical photograph showing injection of the opposite side of case no 3 group III

D. Injection Frequency Protocol: repeated the procedure two times, at one-month interval. At each appointment, the joints were palpated for noise and pain, the affected muscles for pain. The range of jaw motion also was measured interincisally.

\section{III- Postoperative clinical assessment included:}

\section{Subjective evaluation :}

1. Visual Analogue Scale (pain - tenderness chewing). Figure (14)

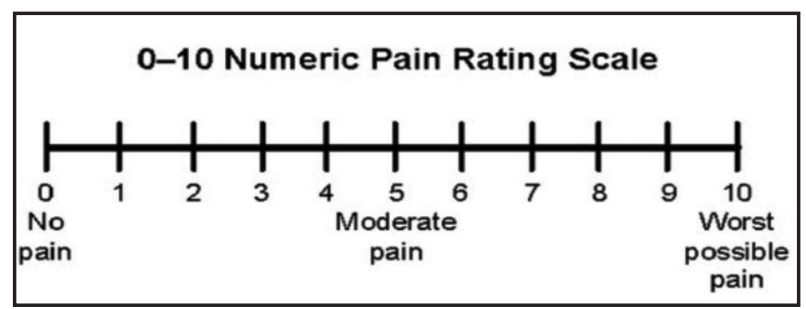

Fig. (14) Showing numeric pain rating scale, visual analogue scale (VAS)

\section{Objective evaluation:}

1. Interincisal opening / PROM/AROM (passive/ active range of motion). Figure (15a-b)

2. Mandibular deviation.

3. Presence or absence of clicking 


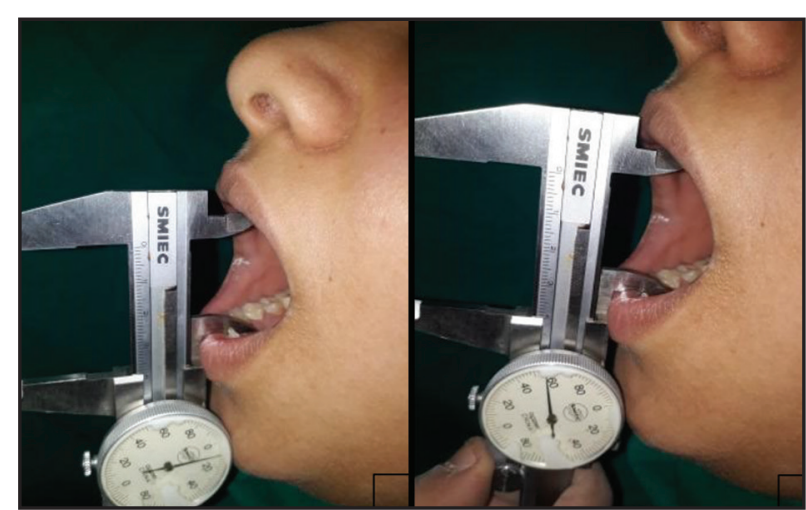

Fig. (15) a- Clinical photograph showing prost-operative clinical assessment of TMJ , PROM in case no 1 group 3, $38 \mathrm{~mm}$, b- Clinical photograph showing postoperative clinical assessment of TMJ , AROM in case no 1 group 3, $40 \mathrm{~mm}$

- Data was tabulated and statistically analyzed.

\section{Statistical Analysis}

Kolmogorov-Smirnov and Shapiro-Wilk tests were used to check the normality.

Repeated measures ANOVA test was used for parametric data, to compare between the three groups as well to study the changes by time within each group. Tukey's post-hoc test was used for pairwise comparisons when ANOVA test is significant.

For non-parametric data, Kruskal-Wallis test was used to compare between the three groups. Friedman's test was used to study the changes after treatment in each group. Dunn's test was used for pair-wise comparisons.

Qualitative data were presented as frequencies and percentages. Fisher's Exact test was used to compare between the three groups.

Friedman's test followed by Dunn's test was done to measures the changes that occurs by times in qualitative data.

\section{Results : follow up}

Follow up was done for 12 months at the following intervals (1, 3, 6 and 12 months) from the last injection.

\section{Base line characteristics}

There was no difference between difference between different base line characteristics (Age, gender, affected TMJ as well as disk displacement) in the three groups.

Maximum Inter-incisal Opening (MIO), Assisted Inter-incisal Opening (AIO) showed parametric distribution

\section{A. Maximum Inter-incisal Opening (MIO)}

\section{Comparison between the three groups}

Pre-operatively, Group II showed the highest mean MIO. Group III showed statistically significantly lower mean value. Group I showed the lowest mean MIO.

After 1 month, no difference between MIO in three groups.

After 3, 6 as well as 12 months, there was no significant difference between Group I and Group III; both showed highest mean MIO values. Group II showed the lowest mean MIO, figure (16).

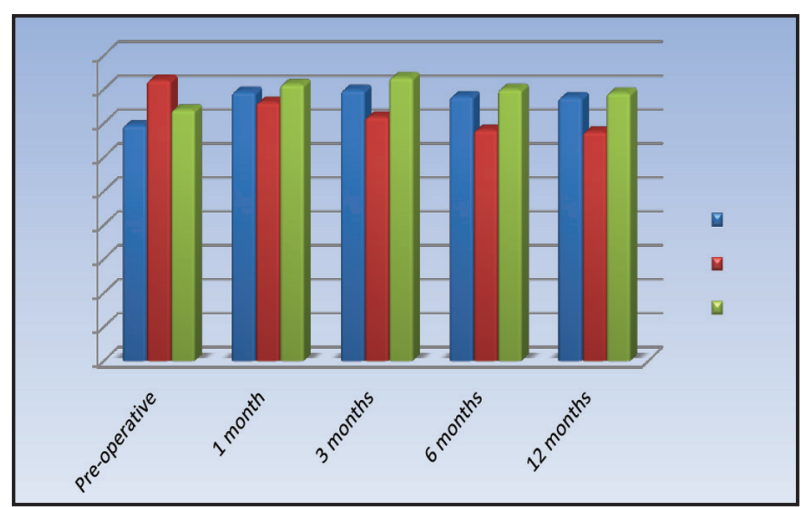

Fig. (16) Bar chart representing comparison between mean MIO in the three groups 


\section{Changes after treatment within each group}

In Group I as well as Group III; there was an increase in $\mathrm{MIO}$ after 1 month. There was no change in mean MIO from 1 to 3,3 to 6 as well as from 6 to 12 months. The mean MIO after 12 months showed statistically significantly higher mean value compared to pre-operative.

While in Group II; decrease in mean MIO after 1 month as well as from 1 to 3 months. No change in mean MIO from 3 to 6 as well as from 6 months to 12 months. The mean MIO after 12 months showed lower mean value compared to preoperative measurement, table (1).

Table (1): The mean, standard deviation (SD) values and results of repeated measures ANOVA test for comparison between $\mathrm{MIO}(\mathrm{mm})$ at different time periods within each group

\begin{tabular}{|c|c|c|c|c|c|c|}
\hline \multirow{2}{*}{ Time } & \multicolumn{2}{|c|}{ Group I } & \multicolumn{2}{|c|}{ Group II } & \multicolumn{2}{|c|}{ Group III } \\
\hline & Mean & $\mathrm{SD}$ & Mean & SD & Mean & SD \\
\hline $\begin{array}{c}\text { Pre- } \\
\text { operative }\end{array}$ & 34.67 & 1.6 & 41.33 & 2.50 & 37.00 & 3.64 \\
\hline 1 month & 39.60 & 2.2 & 38.07 & 2.22 & 40.73 & 4.32 \\
\hline 3 months & 39.87 & 2.6 & 35.93 & 2.74 & 41.73 & 4.45 \\
\hline 6 months & 38.93 & 2.1 & 34.00 & 1.85 & 40.00 & 4.94 \\
\hline 12 months & 38.80 & 2.0 & 33.73 & 1.49 & 39.47 & 4.61 \\
\hline$P$-value & \multicolumn{2}{|c|}{$<0.001 *$} & \multicolumn{2}{|c|}{$<0.001 *$} & \multicolumn{2}{|c|}{$<0.001 *$} \\
\hline
\end{tabular}

*: Significant at $P \leq 0.05$, Different superscripts in the same column are statistically significantly different

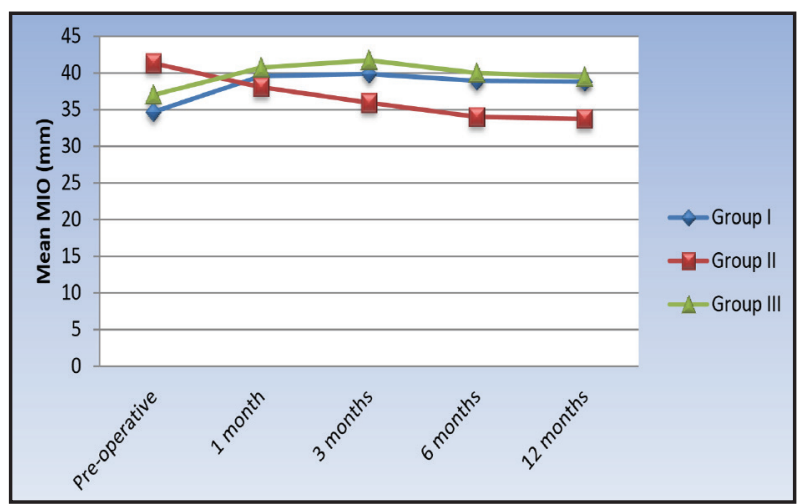

Fig. (17) Line chart representing comparison between mean $\mathrm{MIO}$ at different time periods in each group

\section{B. Assisted Inter-incisal Opening (AIO)}

Pre-operatively, Group II showed the highest mean AIO. Group III showed lower mean value. Group I showed the statistically significantly lowest mean AIO.

After 1 month, no difference between AIO in the three groups.

After 3, 6 as well as 12 months, no difference between Group I and III; both showed the highest mean AIO values. Group II showed the lowest mean AIO

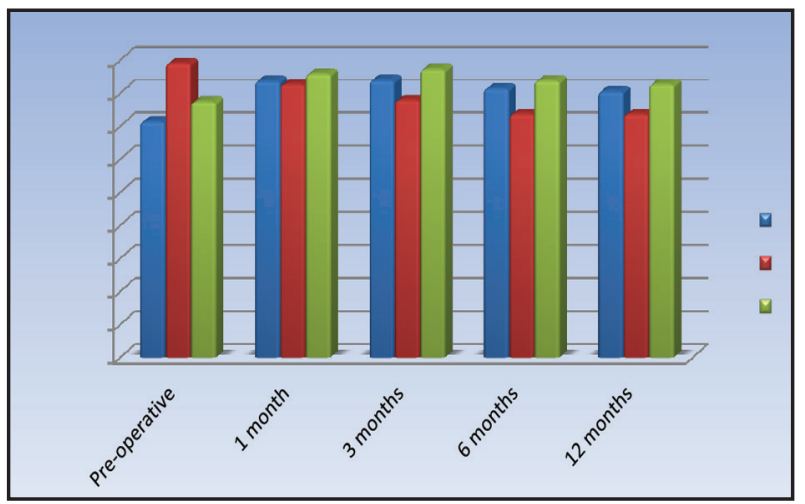

Fig. (18) Bar chart representing comparison between mean AIO in the three groups 


\section{Pain (VAS scores)}

\section{Comparison between the three groups}

Pre-operatively, after 1 month as well as after 3 months; no difference between VAS scores in the three groups.

After 6 as well as 12 months, no difference between Group I and III; both showed the highest mean VAS scores. Group II showed the lowest mean VAS scores.

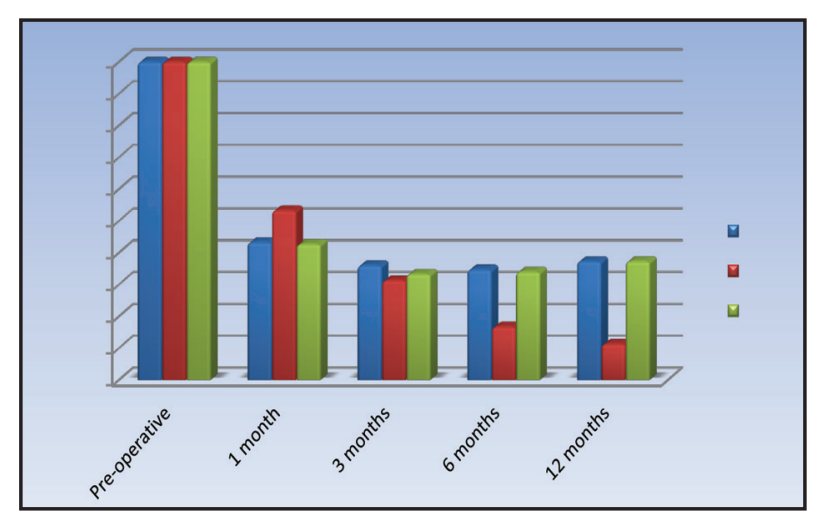

Fig. (19) Bar chart representing comparison between mean VAS scores in the three groups

\section{Deviation Comparison between the three groups}

Pre-operatively, all cases showed deviation.

After 1 month, difference between the three groups. Group II showed the highest prevalence of deviation followed by Group III while Group I showed the lowest prevalence of deviation.

After 3 months, no difference between prevalence of deviation in the three groups.

After 6 months, difference between the three groups. Group III showed the highest prevalence of deviation followed by Group I while Group II showed no deviation.
After 12 months, there was a difference between the three groups. Group I showed the highest prevalence of deviation followed by Group III while Group II showed no deviation.

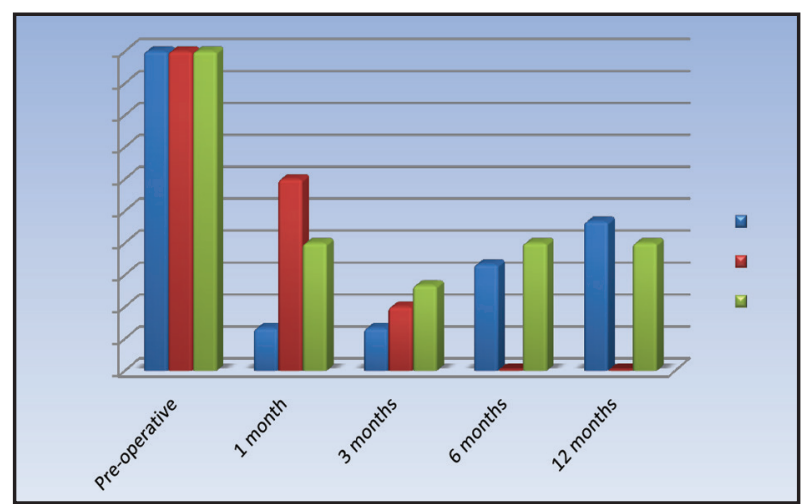

Fig. (20) Bar chart representing prevalence of deviation in the three groups

\section{E. Clicking Comparison between the three groups}

Through all follow up periods, no difference between the three groups.

\section{DISCUSSION}

PRP injection is considered a minimally invasive surgical technique, no complications with PRP injection, also could be done in outpatient clinic. It has lower in various side effects in comparison to with NSAID and TMJ arthrocentesis ${ }^{(3,4)}$.

The purpose of the current study was designed to evaluate the clinical effect of intra-articular injections of PRP for management of internal derangement of TMJ through comparative study with dextrose prolotherapy.

On the current study, in Group I as well as Group III; there was an increase in MIO after 1 month. no change in mean MIO from 1 to 3, 3 to 6 as well as from 6 months to 12 months. The mean MIO after 12 months showed statistically significantly 
higher mean value compared to pre-operative measurement.

These findings were in agreement with a retrospective analysis of patients who received dextrose prolotherapy to TMJ. Improvements in their pain, crunching, and stiffness levels since the last treatment session ${ }^{(5)}$.

This was in agreement with study done in 2015 by Hanci et al ${ }^{(4)}$, on their clinical study on 20 patients for a total of 32 joints with reducible anterior disc displacement , as confirmed by (MRI), were divided into two groups.. Their study showed that intra-articular PRP injection was more effective than arthrocentesis for treatment of anterior disc displacement with reduction.

The outcomes of the current study pain intensity showed significant difference among each group, in Group I and III; a decrease in VAS scores after 1 month as well as from 1 month to 3 months. The mean VAS scores after 12 months showed statistically significantly lower mean score compared to pre-operative measurement. This was in agreement with study done by Hauser et al, who announce in their study that prolotherapy play effective role in decreasing pain and relieving as well the symptoms ${ }^{(5)}$.

While in Group II; a decrease in mean VAS scores after 1 month, from 1 to 3 as well as from 3 to 6 months. The mean VAS scores after 12 months showed statistically significantly lower mean value compared to pre-operative measurement. This was in agreement with a study that showed that PRP injection was effective in reducing the VAS scores and increased the $\mathrm{MIO}^{(7)}$.

In another study, that compare the intensity of pain following intra-articular PRP injection and concluded that PRP injections has a positive impact on the reduction of the intensity of pain experienced by patients treated for temporomandibular joint dysfunction ${ }^{(8)}$. Although, Machon et al, ${ }^{(5)}$ proved in his study that PRP was an effective option for (OA).

The results of this study was in agreement with other researchers who studying PRP treatments highlight the efficiency of this method of management of musculoarticular disorders and its safety as well as the low costs of treatment ${ }^{(10-16)}$.

In the present study, all cases showed deviation preoperatively. However, after 1 month, there was a difference between the three groups. Group II showed the highest prevalence of deviation followed by Group III while Group I showed the lowest prevalence of deviation.

After 3 months, the prevalence of deviation in the three groups showed no difference. After 6 months, difference resulted between the three groups. Group III showed the highest prevalence of deviation followed by group I while group II showed no deviation. After 12 months, significant difference between the three groups. Group I showed the highest prevalence of deviation followed by group III while group II showed no deviation. In group I; there was a significant change in prevalence of deviation by time. There was a decrease in prevalence of deviation after 1 month it increased and no change from 1 month to 3 months. In prevalence of deviation from 3 to 6 as well as from 6 months to 12 months.

In group II; where PRP was injected twice, change in prevalence of deviation by time. There was a decrease in prevalence of deviation after 1 month, from 1 to 3 months as well as from 3 to 6 months. There was no deviation after 6 as well as 12 months which was in agreement with several studies where PRP was injected into the affected TMJs mostly 3 at an interval of 3-5 weeks ${ }^{(17-21)}$. 
In the current study, through all follow up periods clicking changes by time in all three groups. In group I and II; a decrease in prevalence of clicking and this was in agreement with many studies that showed improvement of clicking after one injection of PRP ${ }^{(9,8,22)}$, however other studies showed less improvement who found PRP was effective in the improvement of TMJ pain and restricted mouth opening, however it wasn't so effective in improving the clicking ${ }^{(23,24)}$.

In group III; also there was a decrease in prevalence of clicking and this was in agreement with several studies who conclude that prolotherapy was an efficient, simple and conservative method to treat Temporo-mandibular disorders such as joint pain, sounds, popping and clicking, hypermobility and locking ${ }^{(25,26)}$.

In our study, $1 \mathrm{ml}$ PRP injection once in group I and twice in group II at one month interval was in agreement with Refai et al, ${ }^{(27)}$ who revealed that average volume of joint space was between 1-2 ml however, in many other studies PRP treatment was inconsistent and varied greatly in the injection doses and times including $0.5 \mathrm{ml}$ once ${ }^{(8)}, 0.6 \mathrm{ml}$ once ${ }^{(6)}$ , $1 \mathrm{ml}$ once every 2 weeks twice ${ }^{(9)}, 1.5-2 \mathrm{ml}$ once a month , twice ${ }^{(28)}, 1 \mathrm{ml}$ once every week 3 times ${ }^{(29)}$ and $1 \mathrm{ml}$ once a month 4 times ${ }^{(30)}$ but none of them provided an explanation regarding their selection doses and frequencies.

\section{CONCLUSION AND RECOMMENDATIONS}

PRP and Prolotherapy are successful techniques for management of symptoms associated with the internal derangement of the TMJ as both techniques showed significant improvement in non-assisted mouth opening and significant decrease in pain intensity.

Double intra-articular injection of PRP did not showed any improvement in comparison to single injection other than the deviation, however no improvement in MIO or pain intensity. Further studies, longer observation period and larger samples may be needed to determine the validity of both techniques among different point of study.

\section{REFERENCES}

1. Hall HD. Intra-articular disc displacement Part II, Its significant role in temporomandibular joint pathology. J Oral Maxillofac Surg 1995;53: 1073.

2. Dolwick MF, Katzberg RW, Helms CA. Internal derangements of the temporomandibular joint: fact or fiction? J Prosthet Dent 1983; 49 :415-418.

3. Sun Y, Feng Y, Zhang CQ, Chen SB, Cheng XG. The regenerative effect of platelet-rich plasma on healing in large osteochondral defects. Int Orthop 2010; 34: 589-597.

4. Hanci M, Karamese M, Tosun Z, Aktan TM, Duman $\mathrm{S}$,Savaci N. intraarticular platelet rich plasma injection for the treatment of temporomandibular disorders and a comparison with arthrocentesis. J Craniomaxillofac Surg 2015; 43: 162-166.

5. Machon V, Rehorova M, Sedy J, Foltan R. PlateletRich Plasma in Temporomandibular Joint Osteoarthritis Therapy: A 3-Month Follow-Up Pilot Study 2013; 2: 2.

6. Al-Ani MZ. Stabilisation splint therapy for temporomandibular pain dysfunction syndrome. Cochrane Rev 2007;110-115.

7. Krump E, Nikitas K. Grinstein S: Induction of tyrosine phosphorylation and $\mathrm{Na}+\mathrm{H}+$ exchanger activation during shrinkage of human neutrophils. J Biol Chem 1997; 272:17303-17311.

8. Quinn JH, Bazan NG Identification of prostaglandin E2 and leukotriene B4 in the synovial fluid of painful, dysfunctional temporomandibular joints. J Oral Maxillo fac Surg 1990; 48:968.

9. Sanders B. Arthroscopic surgery of the temporomandibular joint: treatment of internal derangement with persistent closed lock. Oral Surg 1986; 62:361-364.

10. Ra'ed M, Al-Delayme A, Alnuamy S, Hamid F, Azzamily T, Ismaeel S et al. The Efficacy of Platelets Rich Plasma Injection in the Superior Joint Space of the 
Tempromandibular Joint Guided by Ultra Sound in Patients with Non-reducing Disk Displacement. J. Maxillofac Oral Surg 2016; 911-919.

11. Cerza F, Carn S, Carcangiu A. Comparison between hyaluronic acid and platelet-rich plasma, intra-articular infiltration in the treatment of gonarthrosis. Ame J Sport Med 2012; 40: 2822-2827.

12. Albanese A, Licata ME, Polizzi B, and Campisi G. Platelet rich plasma (PRP) in dental and oral surgery : from the wound healing of bone regeneration, Immun and Age 2013; 10: 23-29.

13. Cavallo C, Filardo G, Mariani E. Comparison of platelet rich plasma formulations for cartilage healing: an invitro study. J Bone Joint Surg Amer 2014; 96:423- 429.

14. Bielecki TM, Gazdzik TS, Arendt J, Szczepanski T, Krol W, Wielkoszy T. Antibacterial effect of autologous platelet gel enriched with growth factors and other active substances. Bone Joint J 2007; 89:417-420.

15. Fallouh L, Nakagawa K, Sasho T. Effects of autologous plateletrich plasma on cell viability and collagen synthesis in injured human anterior cruciate ligament, J Bon Joint Surg Ame 2010; 92: 2909-2916.

16. Civinini R, Macera A, Nistri L, Redl B, Innocenti M. The use of autologous blood-derived growth factors in bone regeneration. Clinic Cases Min Bon Metab 2011; 8: 25-31.

17. Sanchez M, Anitua E, Azofra J, Aguirre JJ, Andia I. Intraarticular injection of an autologous preparation rich in growth factors for the treatment of knee OA: a retrospective cohort study. Clin Exp Rheumatol 2008; 26: 910-913.

18. Li M, Zhang C, Ai Z, Yuan T, Feng Y. Therapeutic effectiveness of intra-knee-articular injection of platelet-rich plasma on knee articular cartilage degeneration. Blood Transfus 2011; 25: 1192-1196.

19. Napolitano M, Matera S, Bossio M, Crescibene A, Costabile E. Autologous platelet gel for tissue regeneration in degenerative disorders of the knee. Blood Transfus 2012;10: 72-77.

20. Sanchez M, Guadilla J, Fiz N, Andia I. Ultrasound-guided platelet-rich plasma injections for the treatment of osteoarthritis of the hip. Rheumatol 2012; 51: 144-150.
21. Filardo G, Kon E, Buda R, Timoncini A, Di Martino A. Platelet-rich plasma intra-articular knee injections for the treatment of degenerative cartilage lesions and osteoarthritis. Knee Surg Sports Traumatol Arthrosc 2011;19: 528-535.

22. Kon E, Mandelbaum B, Buda R, Filardo G, Delcogliano M. Platelet rich plasma intra-articular injection versus hyaluronic acid viscosupplementation as treatments for cartilage pathology: from early degeneration to osteoarthritis. Arthroscopy 2011; 27: 1490-1501.

23. Van Osch GJ, Bernsen MR, van Buul GM, Koevoet WL, Kops N, Bos PK, Verhaar JA, et al. Platelet-rich plasma releasate inhibits inflammatory processes in osteoarthritic chondrocytes. Am J Sports Med 2011; 39:2362-2370.

24. Moon S, Lee S, Ryu J. Ultrasound-guided Platelet-rich Plasma Prolotherapy for Temporomandibular Disorders. J Oral Med Pain 2014; 39: 140-145.

25. Hassan E, Ali T, Abdulla N. The clinical efficiency of platelet rich plasma in the treatment of Temporo-mandibular joint disorders. Alexa Dent J 2016; 41: 226-231.

26. Ungor C, Atasoy KT, Taskesen F, Cezairli B, Dayisoylu EH, Tosun E, Senel FC. Short-term results of prolotherapy in the management of temporomandibular joint dislocation. J Craniofac Surg 2013;24:411-415.

27. Refai H, Altahhan O, Elsharkawy R. The efficacy of dextrose prolotherapy for temporomandibular joint hypermobility: a preliminary prospective, randomized, doubleblind, placebo-controlled clinical trial. J Oral Maxillofac Surg 2011;69:2962-2970.

28. Giacomello M, Giacomello A, Mortellaro C. Temporomandibular joint disorders treated with articular injection: the effectiveness of plasma rich in growth factors-Endoret. J Craniofac Surg 2015; 26:709-713.

29. Hegab AF, Ali HE, Elmasry M. Platelet-rich plasma injection as an effective treatment for temporomandibular joint osteoarthritis. J Oral Maxillofac Surg 2015; 73:1706-1713.

30. Kilic SC, Gungormus M, Sumbulu MA. Is arthrocentesis plus platelet- rich plasma superior to arthrocentesis alone in the treatment of temporomandibular joint osteoarthritis? a randomized clinical trial. J Oral Maxillofac Surg 2015; 73:1473-1483. 